\title{
Interval Estimation of Stress-Strength Reliability for a General Exponential Form Distribution with Different Unknown Parameters
}

\author{
Nahed A. Mokhlis ${ }^{1}$, Emad J. Ibrahim ${ }^{1} \&$ Dina M. Gharieb ${ }^{1}$ \\ ${ }^{1}$ Department of Mathematics, Ain Shams University, Cairo, Egypt \\ Correspondence: Emad J. Ibrahim, Department of Mathematics, Ain Shams University, Cairo, Egypt. \\ E-mail: emad_j_ibrahim@yahoo.co.uk
}

Received: July 24, 2017 Accepted: August 8, 2017 Online Published: September 21, 2017

doi:10.5539/ijsp.v6n6p60 URL: https://doi.org/10.5539/ijsp.v6n6p60

\begin{abstract}
This paper deals with interval estimation of the stress-strength reliability, when the stress and strength variables follow a general exponential form distribution. The distribution parameters of both the stress and the strength are assumed to be unknown. Interval estimation for reliability is discussed, using different approaches. The results obtained are applicable to many well known distributions. For illustration of the general results obtained a simulation study is performed with application on Weibull distribution. Numerical comparison of the interval estimators is carried out based on average length, probability coverage, and tail errors.
\end{abstract}

Keywords: Fisher information matrix, Generalized variable method, Bootstrap method, Gibbs sampling, MetropolisHastings algorithm

Math Subject Classification: 62F15; 62F40; 62F25; 62N02.

\section{Introduction}

The stress-strength reliability can be expressed as $\mathrm{R}=\mathrm{P}\left(\mathrm{X}_{1}<\mathrm{X}_{2}\right)$, where the random variables $\mathrm{X}_{1}$ and $\mathrm{X}_{2}$ represent a random stress and a random strength, respectively. The first papers with $\mathrm{P}\left(\mathrm{X}_{1}<\mathrm{X}_{2}\right)$ in their title were introduced by (Birnbaum, 1956), and (Birnbaum and McCarty, 1958). Because of the importance of what we talk about there are a lot of research, which took this topic in different ways and different distributions. One of these ways the point estimation of R, (see, (Saracoglu et al., 2009), and (Panahi and Asadi, 2010)). In many situations knowing an interval estimator is better than just knowing a point estimator because the interval estimator covers the unknown parameter $\mathrm{R}$ with a specified probability, confidence coefficient. Many authors have studied the interval estimation of R. Among them, (Kundu and Gupta, 2006), (Krishnamoorthy and Lin, 2010), (Asgharzadeh et al., 2011), and (Asgharzadeh et al., 2013). (Kotz et al., 2003) have comprehensively covered the problem of point and interval estimation of R. (Singh et al., 2015), and (Asgharzadeh et al., 2017) have also discussed the problem of point and interval estimation of R. Recently, (Mokhlis et al., 2017) introduced point and interval estimation of $R=P\left(X_{1}<X_{2}\right)$ by different methods when $X_{1}$ and $X_{2}$ follow a general exponential form or a general inverse exponential form with survival functions given by either

$$
\overline{\mathrm{F}}_{\mathrm{X}}(\mathrm{x} ; \theta, \mathrm{c})=\exp \left[-\theta \mathrm{g}_{1}(\mathrm{x} ; \mathrm{c})\right]
$$

or

$$
\overline{\mathrm{F}}_{\mathrm{X}}(\mathrm{x} ; \eta, \mathrm{c})=\exp \left[-\eta \mathrm{g}_{2}(\mathrm{x} ; \mathrm{c})\right],
$$

respectively, where $\mathrm{g}_{1}(\mathrm{x} ; \mathrm{c})$ is free from the unknown parameter $\theta$, continuous, monotone increasing, and differentiable function, with $\mathrm{g}_{1}(\mathrm{x} ; \mathrm{c}) \rightarrow 0$ as $\mathrm{x} \rightarrow 0$ and $\mathrm{g}_{1}(\mathrm{x} ; \mathrm{c}) \rightarrow \infty$ as $\mathrm{x} \rightarrow \infty$, while $\mathrm{g}_{2}(\mathrm{x} ; \mathrm{c})$ is free from the unknown parameter $\eta$, continuous, monotone decreasing, and differentiable function, with $\mathrm{g}_{2}(\mathrm{x} ; \mathrm{c}) \rightarrow \infty$ as $\mathrm{x} \rightarrow 0$ and $\mathrm{g}_{2}(\mathrm{x} ; \mathrm{c}) \rightarrow 0$ as $\mathrm{x} \rightarrow \infty$, and $\mathrm{c}$ is a known parameter.

In this paper, we discuss the interval estimation of $\mathrm{R}=\mathrm{P}\left(\mathrm{X}_{1}<\mathrm{X}_{2}\right)$, where $\mathrm{X}_{1}$ and $\mathrm{X}_{2}$ follow a general exponential form distribution. Different methods of estimation are discussed. We obtain an approximate confidence interval of $\mathrm{R}$ via the maximum likelihood estimator of $\mathrm{R}$. Using the generalized variable approach, a generalized confidence interval of $\mathrm{R}$ is presented. Two bootstrap confidence intervals (percentile and t-boot) of $\mathrm{R}$ are also obtained. Bayesian credible interval of $\mathrm{R}$ is also considered, using Markov chain Monte Carlo method (MCMC) in two scenarios. In the first scenario we apply gamma priors, while in the second one we apply gamma and uniform priors. The different interval estimators are illustrated using Weibull distribution, and a comparison is performed among the estimators obtained, on the basis of average length, average coverage probability, and tail errors. 
This paper is organized as follows: In Section 2, the stress-strength reliability, R, and its maximum likelihood estimator are introduced. The approximate confidence interval of $\mathrm{R}$ is considered, in Section 3. In Section 4, the generalized confidence interval of $\mathrm{R}$ is presented, while in Section 5, the percentile and t-bootstrap intervals of $\mathrm{R}$ are obtained. In Section 6, the Bayesian credible intervals of $\mathrm{R}$ are introduced. In Section 7, an illustrative example of the obtained interval estimators of $\mathrm{R}$ is given by using the Weibull distribution. Then a numerical comparison of the intervals obtained is applying the Weibull distribution, in Section 8.

\section{Maximum Likelihood Estimator of $\mathbf{R}$}

Let $X_{1}$ and $X_{2}$ be non-negative independent and continuous random variables, having general exponential form distributions, with the survival functions (SF) and the probability density functions (pdf) given by

$$
\overline{\mathrm{F}}_{\mathrm{X}_{\mathrm{i}}}\left(\mathrm{x} ; \mathrm{b}_{\mathrm{i}}, \mathrm{c}_{\mathrm{i}}\right)=\exp \left[-\varphi_{\mathrm{i}}\left(\mathrm{b}_{\mathrm{i}}, \mathrm{c}_{\mathrm{i}}\right) \mathrm{g}\left(\mathrm{x} ; \mathrm{c}_{\mathrm{i}}\right)\right],
$$

and

$$
\mathrm{f}_{\mathrm{X}_{\mathrm{i}}}\left(\mathrm{x} ; \mathrm{b}_{\mathrm{i}}, \mathrm{c}_{\mathrm{i}}\right)=\varphi_{\mathrm{i}}\left(\mathrm{b}_{\mathrm{i}}, \mathrm{c}_{\mathrm{i}}\right) \mathrm{g}^{\prime}\left(\mathrm{x} ; \mathrm{c}_{\mathrm{i}}\right) \exp \left[-\varphi_{\mathrm{i}}\left(\mathrm{b}_{\mathrm{i}}, \mathrm{c}_{\mathrm{i}}\right) \mathrm{g}\left(\mathrm{x} ; \mathrm{c}_{\mathrm{i}}\right)\right] ; \mathrm{i}=1,2 .
$$

where $\varphi_{i}\left(b_{i}, c_{i}\right)$ is a differentiable function of the unknown parameters $b_{i} \in B_{i}$, and $c_{i} \in \complement_{i}$, and $B_{i}, \complement_{i}$ are the parametric spaces of $b_{i}$, and $c_{i}$, respectively. The function $g\left(x ; c_{i}\right)$ is continuous, monotone increasing, differentiable function such that, $\mathrm{g}\left(\mathrm{x} ; \mathrm{c}_{\mathrm{i}}\right) \rightarrow 0$ as $\mathrm{x} \rightarrow 0$ and $\mathrm{g}\left(\mathrm{x} ; \mathrm{c}_{\mathrm{i}}\right) \rightarrow \infty$ as $\mathrm{x} \rightarrow \infty$, and $\mathrm{g}^{\prime}\left(\mathrm{x} ; \mathrm{c}_{\mathrm{i}}\right)$ is the first derivative of $\mathrm{g}\left(\mathrm{x} ; \mathrm{c}_{\mathrm{i}}\right)$ w.r.t $\mathrm{x}$. Notice that, if $\overline{\mathrm{F}}_{\mathrm{X}_{\mathrm{i}}}\left(\mathrm{x} ; \mathrm{b}_{\mathrm{i}}, \mathrm{c}_{\mathrm{i}}\right)$ is defined on $(\alpha, \beta)$, then $\mathrm{g}\left(\mathrm{x} ; \mathrm{c}_{\mathrm{i}}\right) \rightarrow 0$ as $\mathrm{x} \rightarrow \alpha^{+}$and $\mathrm{g}\left(\mathrm{x} ; \mathrm{c}_{\mathrm{i}}\right) \rightarrow \infty$ as $\mathrm{x} \rightarrow \beta^{-}$. Then the stress-strength reliability is given by

$$
\mathrm{R}=\mathrm{P}\left(\mathrm{X}_{1}<\mathrm{X}_{2}\right)=\int_{0}^{\infty} \varphi_{1} \mathrm{~g}^{\prime}\left(\mathrm{z} ; \mathrm{c}_{1}\right) \exp \left(-\sum_{\mathrm{i}=1}^{2} \varphi_{\mathrm{i}} \mathrm{g}\left(\mathrm{z} ; \mathrm{c}_{\mathrm{i}}\right)\right) \mathrm{dz},
$$

where $\varphi_{\mathrm{i}}=\varphi_{\mathrm{i}}\left(\mathrm{b}_{\mathrm{i}}, \mathrm{c}_{\mathrm{i}}\right) ; \mathrm{i}=1$, 2. In case of $\mathrm{c}_{1}=\mathrm{c}_{2}=\mathrm{c}$, the stress-strength reliability can be expressed as $\mathrm{R}=\frac{\varphi_{1}}{\varphi_{1}+\varphi_{2}}$ as in (Mokhils et al., 2017).

If $\underline{\mathbf{X}}_{\mathrm{i}}=\left(\mathrm{X}_{\mathrm{i} 1}, \mathrm{X}_{\mathrm{i} 2}, \ldots, \mathrm{X}_{\mathrm{in}_{\mathrm{i}}}\right) ; \mathrm{i}=1,2$, are two independent random samples from populations with distributions given by (1), then the likelihood function is given as

$$
\mathrm{L}\left(\underline{x}_{1}, \underline{x}_{2} \mid \theta\right)=\exp \left[\sum_{i=1}^{2} n_{i} \ln \varphi_{i}\left(b_{i}, c_{i}\right)+\sum_{i=1}^{2} \sum_{j=1}^{n_{i}} \ln g^{\prime}\left(x_{i j} ; c_{i}\right)-\sum_{i=1}^{2} \varphi_{i}\left(b_{i}, c_{i}\right) \sum_{j=1}^{n_{i}} g\left(x_{i j} ; c_{i}\right)\right],
$$

where $x_{i j}$ is the $\mathrm{j}^{\text {th }}$ observation in the sample $\underline{\mathbf{X}}_{\mathbf{i}} ; \mathrm{j}=1, \ldots, \mathrm{n}_{\mathrm{i}}, \mathrm{i}=1,2$, and $\theta=\left(c_{1}, c_{2}, b_{1}, b_{2}\right)$. The log-likelihood function is

$$
\ln \mathrm{L}\left(\underline{x}_{1}, \underline{x}_{2} \mid \theta\right)=\sum_{i=1}^{2} n_{i} \ln \varphi_{i}\left(b_{i}, c_{i}\right)+\sum_{i=1}^{2} \sum_{j=1}^{n_{i}} \ln g^{\prime}\left(x_{i j} ; c_{i}\right)-\sum_{i=1}^{2} \varphi_{i}\left(b_{i}, c_{i}\right) \sum_{j=1}^{n_{i}} g\left(x_{i j} ; c_{i}\right) .
$$

Partially differentiating $\ln \mathrm{L}\left(\underline{x}_{1}, \underline{x}_{2} \mid \theta\right)$ with respect to $\theta$, and equating to 0 , we get

$$
\frac{\partial \ln \mathrm{L}}{\partial b_{i}}=\left(\frac{n_{i}}{\varphi_{i}}-\sum_{j=1}^{n_{i}} g\left(x_{i j} ; c_{i}\right)\right) \frac{\partial \varphi_{\mathrm{i}}}{\partial b_{i}}=0,
$$

and

$$
\frac{\partial \ln \mathrm{L}}{\partial c_{i}}=\frac{n_{i}}{\varphi_{i}} \frac{\partial \varphi_{i}}{\partial c_{i}}+\sum_{j=1}^{n_{i}} \frac{\partial}{\partial c_{i}} \ln g^{\prime}\left(x_{i j} ; c_{i}\right)-\frac{\partial \varphi_{i}}{\partial c_{i}} \sum_{j=1}^{n_{i}} g\left(x_{i j} ; c_{i}\right)-\varphi_{i} \sum_{j=1}^{n_{i}} \frac{\partial}{\partial c_{i}} g\left(x_{i j} ; c_{i}\right)=0 ; \mathrm{i}=1,2 .
$$

From (6) and knowing that $\frac{\partial \varphi_{\mathrm{i}}}{\partial b_{i}} \neq 0$, the MLEs, $\hat{\varphi}_{\mathrm{i}}$ of $\varphi_{\mathrm{i}}$, are given by

$$
\hat{\varphi}_{i}=\frac{n_{i}}{\sum_{j=1}^{n_{i}} g\left(x_{i j} ; \hat{c}_{i}\right)} ; \mathrm{i}=1,2 .
$$

The MLEs $\hat{c}_{i}$ of $c_{i}$ can be obtained, by substituting $\hat{\varphi}_{\mathrm{i}}$ into (7) and solving numerically. Once we obtain $\hat{c}_{\mathrm{i}}$, the MLEs, $\hat{\varphi}_{i}$ of $\varphi_{i}$ are completely determined by substituting $\hat{c}_{i}$ in (8). Using the invariance property, the MLEs, $\hat{b}_{i}$ of $b_{i}$ can be deduced from $\hat{\varphi}_{\mathrm{i}}\left(\hat{\mathrm{b}}_{\mathrm{i}}, \hat{\mathrm{c}}_{\mathrm{i}}\right) ; \mathrm{i}=1$, 2. The corresponding MLE, $\hat{\mathrm{R}}$ of $\mathrm{R}$ can be obtained by replacing the parameters in (3) with their MLEs. 


\section{Approximate Confidence Interval of $\mathrm{R}$ (ACI)}

As we know from the asymptotic maximum likelihood properties, the approximate $(1-\alpha) 100 \%$ confidence interval for $\mathrm{R}$ is $\left(\hat{\mathrm{R}} \pm \mathrm{Z}_{(1-\alpha / 2)} \sqrt{\hat{\sigma}_{\hat{\mathrm{R}}}^{2}}\right)$, where $\mathrm{z}_{(1-\alpha / 2)}$ is the $(1-\alpha / 2)$ th quantile of the standard normal distribution and $\hat{\sigma}_{\hat{\mathrm{R}}}^{2}$ is the estimator of variance of $\hat{\mathrm{R}}, \hat{\sigma}_{\hat{\mathrm{R}}}^{2}=\left.\mathrm{A}^{\mathrm{t}} \mathrm{B}^{-1} \mathrm{~A}\right|_{\theta=\hat{\theta}}$, where $\hat{\theta}$ is the MLE of $\theta, \mathrm{B}^{-1}$ is the inverse of the Fisher information matrix $\mathrm{B}$ of $\theta, \mathrm{A}^{\mathrm{t}}$ is the transpose of matrix A, (see, (Rao, 1965)), where

$$
\begin{gathered}
B=-E\left[\frac{\partial^{2} \ln L}{\partial \theta_{i} \partial \theta_{j}}\right], \quad A=\left[\frac{\partial R}{\partial \theta_{i}}\right] ; i, j=1,2,3,4, \\
\frac{\partial^{2} \ln L}{\partial c_{i}^{2}}=-\frac{n_{i}}{\varphi_{i}^{2}}\left(\frac{\partial \varphi_{i}}{\partial c_{i}}\right)^{2}+\sum_{j=1}^{n_{i}} \frac{\partial^{2}}{\partial c_{i}^{2}} \ln g^{\prime}\left(x_{i j} ; c_{i}\right)-\varphi_{i} \sum_{j=1}^{n_{i}} \frac{\partial^{2}}{\partial c_{i}^{2}} g\left(x_{i j} ; c_{i}\right)-2 \frac{\partial \varphi_{i}}{\partial c_{i}} \sum_{j=1}^{n_{i}} \frac{\partial}{\partial c_{i}} g\left(x_{i j} ; c_{i}\right), \\
\frac{\partial^{2} \ln L}{\partial c_{i} \partial b_{i}}=\frac{\partial^{2} \ln L}{\partial b_{i} \partial c_{i}}=-\left(\frac{n_{i}}{\varphi_{i}^{2}} \frac{\partial \varphi_{i}}{\partial c_{i}}+\sum_{j=1}^{n_{i}} \frac{\partial}{\partial c_{i}} g\left(x_{i j} ; c_{i}\right)\right) \frac{\partial \varphi_{i}}{\partial b_{i}} \\
\frac{\partial^{2} \ln L}{\partial b_{i}^{2}}=-\frac{n_{i}}{\varphi_{i}^{2}}\left(\frac{\partial \varphi_{i}}{\partial b_{i}}\right)^{2} \\
\frac{\partial^{2} \ln L}{\partial c_{i} \partial c_{j}}=\frac{\partial^{2} \ln L}{\partial b_{i} \partial b_{j}}=\frac{\partial^{2} \ln L}{\partial b_{i} \partial c_{j}}=0 ; i \neq j \text { and } \mathrm{i}, \mathrm{j}=1,2 .
\end{gathered}
$$

We can see that the explicit expression of $\sigma_{\hat{\mathrm{R}}}^{2}$ depends on the forms of $\varphi_{\mathrm{i}}, \mathrm{g}^{\prime}\left(\mathrm{x}_{\mathrm{ij}} ; \mathrm{c}_{\mathrm{i}}\right)$ and $\mathrm{g}\left(\mathrm{x}_{\mathrm{ij}} ; \mathrm{c}_{\mathrm{i}}\right) ; \mathrm{j}=1, \ldots, \mathrm{n}_{\mathrm{i}}, \mathrm{i}=1,2$.

\section{Generalized Confidence Interval of R (GCI)}

We obtain the generalized confidence interval for $\mathrm{R}$ by applying the generalized variable approach. The generalized pivotal quantity (GPQ) for $\mathrm{R}, \mathrm{G}_{\mathrm{R}}=\mathrm{R}\left(\mathrm{G}_{\varphi_{1}}, \mathrm{G}_{\varphi_{2}}, \mathrm{G}_{\mathrm{c}_{1}}, \mathrm{G}_{\mathrm{c}_{2}}\right)$ is obtained by replacing the parameters in (3) with $\mathrm{G}_{\varphi_{\mathrm{i}}}=\varphi_{\mathrm{i}}\left(\mathrm{G}_{\mathrm{b}_{\mathrm{i}}}, \mathrm{G}_{\mathrm{c}_{\mathrm{i}}}\right), \mathrm{G}_{\mathrm{b}_{\mathrm{i}}}$, and $\mathrm{G}_{\mathrm{c}_{\mathrm{i}}}$, where $\mathrm{G}_{\varphi_{\mathrm{i}}}, \mathrm{G}_{\mathrm{b}_{\mathrm{i}}}$, and $\mathrm{G}_{\mathrm{c}_{\mathrm{i}}}$ denote the GPQs for $\varphi_{\mathrm{i}}, \mathrm{b}_{\mathrm{i}}$, and $\mathrm{c}_{i} ; \mathrm{i}=1,2$, respectively. The GPQ is a function of observed statistics and random variables whose distribution is free of unknown parameters. The $(1-\alpha) 100 \%$ generalized confidence interval of $\mathrm{R}$ can be obtained as $\left(\mathrm{G}_{\mathrm{R}(\alpha / 2)}, \mathrm{G}_{\mathrm{R}(1-\alpha / 2)}\right)$, where $\mathrm{G}_{\mathrm{R}(\alpha / 2)}$ and $\mathrm{G}_{\mathrm{R}(1-\alpha / 2)}$ are the $(\alpha / 2)$ th and $(1-\alpha / 2)$ th quantiles of R, respectively.

\section{Bootstrap Confidence Intervals of $\mathbf{R}$ (boot)}

The bootstrap confidence interval of $\mathrm{R}$ can be estimated using either percentile bootstrap or t-bootstrap. The bootstrap samples will be generated firstly, using the following bootstrap sampling algorithm, (see, (Efron, 1994)).

\section{Algorithm 1.}

1. Generate $\underline{\mathbf{X}}_{\mathbf{i}} ; \mathrm{i}=1,2$, from (1), and compute the MLEs $\left(\hat{\mathrm{c}}_{1}, \hat{\mathrm{c}}_{2}, \hat{\varphi}_{1}, \hat{\varphi}_{2}, \hat{\mathrm{R}}\right)$ of $\left(\mathrm{c}_{1}, \mathrm{c}_{2}, \varphi_{1}, \varphi_{2}, \mathrm{R}\right)$.

2. Resample two independent random samples $\underline{\mathbf{X}}_{\mathbf{i}}^{* *} ; \mathrm{i}=1,2$, from $\underline{\mathbf{X}}_{\mathrm{i}} ; \mathrm{i}=1,2$, respectively; compute the MLEs $\left(\hat{\mathrm{c}}_{1}^{* *}, \hat{\mathrm{c}}_{2}^{* *}, \hat{\varphi}_{1}^{* *}, \hat{\varphi}_{2}^{* *}, \hat{\mathrm{R}}^{* *}\right)$ of $\left(\mathrm{c}_{1}, \mathrm{c}_{2}, \varphi_{1}, \varphi_{2}, \mathrm{R}\right)$.

3. Repeat Step 2, $N$ times to obtain a set of bootstrap samples of $R$, say $\left\{\hat{R}_{j}^{* *} ; j=1, \ldots, N\right\}$. Order $\hat{R}_{j}^{* *}$, in an increasing order.

4. Construct two different bootstrap intervals of R:

(a) Percentile bootstrap (P-boot)

The $(1-\alpha) 100 \%$ percentile bootstrap confidence interval of $\mathrm{R}$ is $\left(\hat{\mathrm{R}}_{(\alpha / 2)}^{* *}, \hat{\mathrm{R}}_{(1-\alpha / 2)}^{* *}\right)$, where $\hat{\mathrm{R}}_{(\alpha / 2)}^{* *}$ and $\hat{\mathrm{R}}_{(1-\alpha / 2)}^{* *}$ are the $(\alpha / 2)$ th and $(1-\alpha / 2)$ th quantiles of $\mathrm{R}$, respectively.

(b) T-bootstrap (T-boot)

The $(1-\alpha) 100 \% \mathrm{t}$-bootstrap confidence interval of $\mathrm{R}$ is given by $\left(\hat{\mathrm{R}}-\hat{\mathrm{t}}_{(1-\alpha / 2)} \mathrm{S}^{* *}, \hat{\mathrm{R}}-\hat{\mathrm{t}}_{(\alpha / 2)} \mathrm{S}^{* *}\right)$, where $\mathrm{S}^{* *}$ be the sample standard deviation of $\left\{\hat{\mathrm{R}}_{\mathrm{j}}^{* *} ; \mathrm{j}=1, \ldots, \mathrm{N}\right\}$ and $\hat{\mathrm{t}}_{(\alpha)}$ be the $(\alpha)$ th quantile of $\left\{\frac{\hat{\mathrm{R}}_{\mathrm{j}}^{* *}-\hat{\mathrm{R}}}{\mathrm{S}^{* *}} ; \mathrm{j}=1, \ldots, \mathrm{N}\right\}$. 


\section{Bayesian Credible Interval of R (BCI)}

We suggest two different scenarios to estimate the Bayesian credible interval of $\mathrm{R}$ by applying MCMC. For the first scenario, we assume gamma priors for $\varphi_{1}, \varphi_{2}, \mathrm{c}_{1}$, and $\mathrm{c}_{2}$, while in the second scenario, we consider independent gamma priors for $\varphi_{1}, \varphi_{2}$ and uniform priors for $\mathrm{c}_{1}, \mathrm{c}_{2}$ as the available priors information is weak.

\subsection{Gamma Priors $(G-B C I)$}

Let the prior density of $\varphi_{i} ; \mathrm{i}=1,2$ be gamma given by

$$
f_{\varphi_{i}}\left(\varphi_{i}\right)=\frac{\beta_{i}^{\alpha_{i}}}{\Gamma \alpha_{i}} \varphi_{i}^{\alpha_{i}-1} e^{-\beta_{i} \varphi_{i}} ; \quad \varphi_{i}, \alpha_{i}, \beta_{i}>0,
$$

and also assume that $\varphi_{1}$ and $\varphi_{2}$ are independent. Moreover, assume that $\mathrm{c}_{\mathrm{i}} ; \mathrm{i}=1,2$ have gamma priors with probability density functions

$$
f_{c_{i}}\left(c_{i}\right)=\frac{\lambda_{i}^{\delta_{i}}}{\Gamma \delta_{i}} c_{i}^{\delta_{i}-1} e^{-\lambda_{i} c_{i}} ; \quad c_{i}, \delta_{i}, \lambda_{i}>0,
$$

and $c_{1}$ and $c_{2}$ are independent. From (4), (9), and (10), the joint posterior density function of $\varphi_{1}, \varphi_{2}, c_{1}$, and $c_{2}$ can be obtained as

$$
\pi\left(\varphi_{1}, \varphi_{2}, c_{1}, c_{2} \mid \underline{x}_{1}, \underline{x}_{2}\right)=\frac{\mathrm{L}\left(\underline{x}_{1}, \underline{x}_{2} \mid \theta\right) f_{\varphi_{1}}\left(\varphi_{1}\right) f_{\varphi_{2}}\left(\varphi_{2}\right) f_{c_{1}}\left(c_{1}\right) f_{c_{2}}\left(c_{2}\right)}{\int_{0}^{\infty} \int_{0}^{\infty} \int_{0}^{\infty} \int_{0}^{\infty} \mathrm{L}\left(\underline{x}_{1}, \underline{x}_{2} \mid \theta\right) f_{\varphi_{1}}\left(\varphi_{1}\right) f_{\varphi_{2}}\left(\varphi_{2}\right) f_{c_{1}}\left(c_{1}\right) f_{c_{2}}\left(c_{2}\right) d \varphi_{1} d \varphi_{2} d c_{1} d c_{2}} .
$$

Since, the joint posterior density function cannot be obtained analytically, we apply MCMC method to estimate the Bayesian credible interval of R. There are generally two algorithms, the Gibbs sampling and the Metropolis-Hastings algorithm. If the conditional distribution for each parameter is a known distribution, the Gibbs sampling can be used. If the conditional distribution doesn't look like any known distribution, in this case the Metropolis-Hastings algorithm can be useful. To perform the MCMC method, we first find the marginal posterior distributions of $\varphi_{\mathrm{i}}$ and $\mathrm{c}_{\mathrm{i}}$. The marginal posterior distribution of $\varphi_{\mathrm{i}}$ is

$$
\pi_{1}\left(\varphi_{i} \mid c_{i}, \underline{x}_{1}, \underline{x}_{2}\right)=\frac{\left(\beta_{i}+\sum_{j=1}^{n_{i}} g\left(x_{i j} ; c_{i}\right)\right)^{\left(n_{i}+\alpha_{i}\right)}}{\Gamma\left(n_{i}+\alpha_{i}\right)} \varphi_{i}^{\left(n_{i}+\alpha_{i}-1\right)} \exp \left[-\varphi_{i}\left(\beta_{i}+\sum_{j=1}^{n_{i}} g\left(x_{i j} ; c_{i}\right)\right)\right] ; \quad \mathrm{i}=1,2 .
$$

The marginal posterior distribution of $c_{i}$ is

$$
\pi_{1}\left(c_{i} \mid \underline{x}_{1}, \underline{x}_{2}\right)=K_{i}^{-1} \exp \left[\left(\delta_{i}-1\right) \ln c_{i}-\lambda_{i} c_{i}+\sum_{j=1}^{n_{i}} \ln g^{\prime}\left(x_{i j} ; c_{i}\right)-\left(n_{i}+\alpha_{i}\right) \ln \left(\beta_{i}+\sum_{j=1}^{n_{i}} g\left(x_{i j} ; c_{i}\right)\right)\right],
$$

where

$$
K_{i}=\int_{0}^{\infty} \exp \left[\left(\delta_{i}-1\right) \ln c_{i}-\lambda_{i} c_{i}+\sum_{j=1}^{n_{i}} \ln g^{\prime}\left(x_{i j} ; c_{i}\right)-\left(n_{i}+\alpha_{i}\right) \ln \left(\beta_{i}+\sum_{j=1}^{n_{i}} g\left(x_{i j} ; c_{i}\right)\right)\right] d c_{i} ; \mathrm{i}=1,2 .
$$

However, we shall use the union of the Metropolis-Hastings with Gibbs sampling, (see, (Asgharzadeh et al., 2013)). The procedure is shown by the following algorithm.

\section{Algorithm 2.}

1. Choose the starting values $\mathrm{c}_{1}^{(0)}$ and $\mathrm{c}_{2}^{(0)}$.

2. For $\mathrm{j}=1$ to $\mathrm{N}$ times.

3. Generate $\varphi_{\mathrm{i}}^{(\mathrm{j})} ; \mathrm{i}=1,2$, from (11).

4. Generate $c_{i}^{(j)} ; i=1,2$, from (12). Using the Metropolis-Hastings algorithm with the normal proposal distribution $\pi_{\mathrm{i}} \sim \mathrm{N}\left(\mathrm{c}_{\mathrm{i}}^{(\mathrm{j}-1)}, 1\right) ; \mathrm{i}=1,2$.

(a) Generate $\xi_{\mathrm{i}}$ from the proposal distribution $\pi_{\mathrm{i}} ; \mathrm{i}=1,2$, respectively. 
(b) Define $\mathrm{Q}_{\mathrm{i}}=\min \left\{1, \frac{\pi_{1}\left(\underline{\xi}_{i} \mid \underline{\mathrm{x}}_{1}, \underline{\mathrm{x}}_{2}\right) \pi_{\mathrm{i}}\left(\mathrm{c}_{\mathrm{i}}^{(\mathrm{j}-1)}\right)}{\pi_{1}\left(\mathrm{c}_{\mathrm{i}}^{(\mathrm{j}-1)} \mid \underline{\mathrm{x}}_{1}, \underline{\mathrm{x}}_{2}\right) \pi_{\mathrm{i}}\left(\xi_{\mathrm{i}}\right)}\right\} ; \mathrm{i}=1,2$.

(c) Generate $u_{i}$ from Uniform $(0,1)$. Take $c_{i}^{(j)}=\left\{\begin{array}{c}\xi_{i} ; u_{i} \leq Q_{i}, \\ c_{i}^{(j-1)} ; \text { otherwise }\end{array} ; i=1,2\right.$, respectively.

5. Compute the $\mathrm{R}^{\mathrm{j}}$ from (3).

6. End j loop.

7. Repeat the steps $2-6, \mathrm{~N}$ times, and sort $\mathrm{R}^{\mathrm{j}} ; \mathrm{j}=1, \ldots, \mathrm{N}$, ascending.

8. Construct the (1- 1$) 100 \%$ Bayesian credible interval of $\mathrm{R}$ as $\left(\widetilde{\mathrm{R}}_{\mathrm{g}(\alpha / 2)}, \widetilde{\mathrm{R}}_{\mathrm{g}(1-\alpha / 2)}\right)$, where $\widetilde{\mathrm{R}}_{\mathrm{g}(\alpha / 2)}$ and $\widetilde{\mathrm{R}}_{\mathrm{g}(1-\alpha / 2)}$ are the $(\alpha / 2)$ th and $(1-\alpha / 2)$ th quantiles of $\mathrm{R}$, respectively.

\subsection{Mixed Priors (M-BCI)}

Let the prior density function of $\varphi_{\mathrm{i}}$ be as (9); $\mathrm{i}=1,2$, and assume that, $\mathrm{c}_{\mathrm{i}}$ has uniform prior distribution with probability density function

$$
f\left(c_{i}\right)=1 ; c_{i}>0, i=1,2 .
$$

From the likelihood function in (4) and the prior density functions of $\varphi_{1}, \varphi_{2}, c_{1}$, and $c_{2}$, the joint density function can be obtained as

$$
L_{2}\left(\underline{x}_{1}, \underline{x}_{2}, \varphi_{1}, \varphi_{2}, c_{1}, c_{2}\right)=\frac{\beta_{1}^{\alpha_{1}} \beta_{2}^{\alpha_{2}}}{\Gamma \alpha_{1} \Gamma \alpha_{2}} \exp \left[\sum_{i=1}^{2}\left(n_{i}+\alpha_{i}-1\right) \ln \varphi_{i}+\sum_{i=1}^{2} \sum_{j=1}^{n_{i}} \ln g^{\prime}\left(x_{i j} ; c_{i}\right)-\sum_{i=1}^{2} \varphi_{i}\left(\beta_{i}+\sum_{j=1}^{n_{i}} g\left(x_{i j} ; c_{i}\right)\right)\right] .
$$

The joint posterior density functions of $\varphi_{1}, \varphi_{2}, \mathrm{c}_{1}$, and $\mathrm{c}_{2}$ based on (13) is given as

$$
L_{2}\left(\varphi_{1}, \varphi_{2}, c_{1}, c_{2} \mid \underline{x}_{1}, \underline{x}_{2}\right)=\frac{L_{2}\left(\underline{x}_{1}, \underline{x}_{2}, \varphi_{1}, \varphi_{2}, c_{1}, c_{2}\right)}{\int_{0}^{\infty} \int_{0}^{\infty} \int_{0}^{\infty} \int_{0}^{\infty} L_{2}\left(\underline{x}_{1}, \underline{x}_{2}, \varphi_{1}, \varphi_{2}, c_{1}, c_{2}\right) d \varphi_{1} d \varphi_{2} d c_{1} d c_{2}} .
$$

The marginal posterior distribution of $\varphi_{\mathrm{i}}$ is given by the same as in (11), while the marginal posterior distribution of $\mathrm{c}_{\mathrm{i}}$ becomes

$$
\pi_{2}\left(c_{i} \mid \underline{x}_{1}, \underline{x}_{2}\right)=T_{i}^{-1} \exp \left[\sum_{j=1}^{n_{i}} \ln g^{\prime}\left(x_{i j} ; c_{i}\right)-\left(n_{i}+\alpha_{i}\right) \ln \left(\beta_{i}+\sum_{j=1}^{n_{i}} g\left(x_{i j} ; c_{i}\right)\right)\right],
$$

where

$$
T_{i}=\int_{0}^{\infty} \exp \left[\sum_{j=1}^{n_{i}} \ln g^{\prime}\left(x_{i j} ; c_{i}\right)-\left(n_{i}+\alpha_{i}\right) \ln \left(\beta_{i}+\sum_{j=1}^{n_{i}} g\left(x_{i j} ; c_{i}\right)\right)\right] d c_{i} ; \mathrm{i}=1,2 .
$$

As we notice that, the marginal posterior distributions of $c_{1}$ and $c_{2}$ do not have a known form. Using a technique similar to that in Algorithm 2 except for the posterior distribution of $c_{i} ; i=1,2$. The $(1-\alpha) 100 \%$ Bayesian credible interval of $\mathrm{R}$ can be obtained as $\left(\widetilde{\mathrm{R}}_{\mathrm{m}(\alpha / 2)}, \widetilde{\mathrm{R}}_{\mathrm{m}(1-\alpha / 2)}\right)$, where $\widetilde{\mathrm{R}}_{\mathrm{m}(\alpha / 2)}$ and $\widetilde{\mathrm{R}}_{\mathrm{m}(1-\alpha / 2)}$ are the $(\alpha / 2)$ th and (1- $\left.\alpha / 2\right)$ th quantiles of $\mathrm{R}$, respectively.

\section{Illustrative Example}

We see that, the Weibull distribution follows the general exponential form in (1), with $\varphi_{\mathrm{i}}=\frac{1}{\mathrm{~b}_{\mathrm{i}}^{\mathrm{c}_{\mathrm{i}}}}$ and $\mathrm{g}\left(\mathrm{x} ; \mathrm{c}_{\mathrm{i}}\right)=\mathrm{x}^{\mathrm{c}_{\mathrm{i}}}$. If $\underline{\mathbf{X}}_{i} ; \mathrm{i}=1,2$, are two independent random samples from Weibull distributions with the survival function given as $\overline{\mathrm{F}}_{X_{\mathrm{i}}}\left(\mathrm{x} ; \mathrm{b}_{\mathrm{i}}, \mathrm{c}_{\mathrm{i}}\right)=\exp \left[-\frac{1}{\mathrm{c}_{\mathrm{i}}^{\mathrm{i}}} \mathrm{x}^{\mathrm{c}_{\mathrm{i}}}\right] ; \mathrm{i}=1,2$. Then from (3), the stress-strength reliability is given as $\mathrm{R}=\frac{\mathrm{c}_{1}}{\mathrm{~b}_{1}^{1}} \int_{0}^{\infty} \mathrm{z}^{\mathrm{c}_{1}-1} \exp \left[-\sum_{\mathrm{i}=1}^{2} \frac{1}{\mathrm{~b}_{1}^{\mathrm{c}_{\mathrm{i}}}} \mathrm{Z}^{\mathrm{c}_{\mathrm{i}}}\right] \mathrm{dz}$. Using the MLEs, $\left(\hat{\mathrm{c}}_{1}, \hat{\mathrm{c}}_{2}, \hat{\varphi}_{1}, \hat{\varphi}_{2}, \hat{\mathrm{R}}\right)$, the approximate $(1-\alpha) 100 \%$ confidence interval for R can be obtained. Using the Newton-Raphson iterative method, the MLE $\hat{c}_{\mathrm{i}}$, of $\mathrm{c}_{\mathrm{i}}$ from (7) is given by

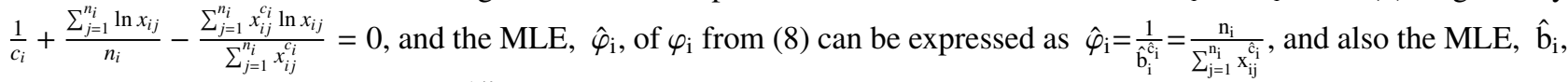

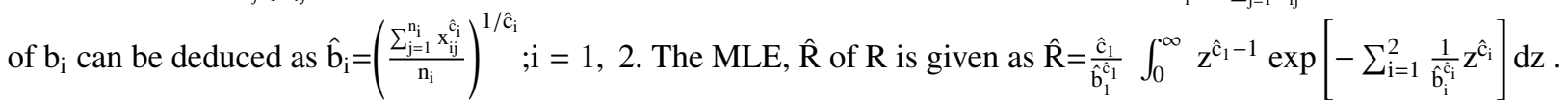


Applying the method in Section 4, we can have $G_{R}$ by replacing $\varphi_{i}$, $b_{i}$ and $c_{i}$ with $G_{\varphi_{i}}, G_{b_{i}}$ and $G_{c_{i}}$, respectively, where

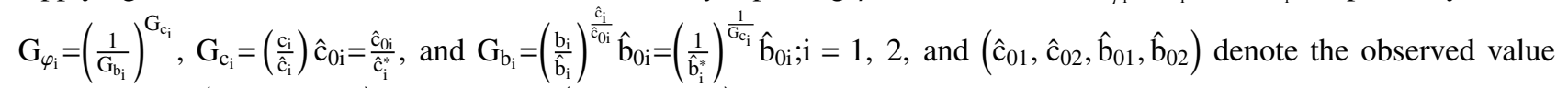
of the MLEs $\left(\hat{c}_{1}, \hat{c}_{2}, \hat{b}_{1}, \hat{b}_{2}\right)$. The MLEs $\left(\hat{c}_{1}^{*}, \hat{c}_{2}^{*}, \hat{b}_{1}^{*}, \hat{b}_{2}^{*}\right)$ of $\left(c_{1}, c_{2}, b_{1}, b_{2}\right)$ are based on two independent samples from standard exponential distributions (see, (Krishnamoorthy et al., 2009), and (Krishnamoorthy and Lin, 2010)). The quantity $\left(\frac{\hat{\mathrm{c}}_{\mathrm{i}}}{\mathrm{c}_{\mathrm{i}}}\right)$ is distributed as $\hat{\mathrm{c}}_{\mathrm{i}}^{*}$ and the quantity $\hat{c}_{\mathrm{i}} \ln \left(\frac{\hat{b}_{\mathrm{i}}}{\mathrm{b}_{\mathrm{i}}}\right)$ is distributed as $\hat{c}_{\mathrm{i}}^{*} \ln \left(\hat{\mathrm{b}}_{\mathrm{i}}^{*}\right) ; i=1,2$, and the distributions of $\left(\frac{\hat{c}_{\mathrm{i}}}{\mathrm{c}_{\mathrm{i}}}\right)$ and $\hat{c}_{\mathrm{i}} \ln \left(\frac{\hat{b}_{\mathrm{i}}}{\mathrm{b}_{\mathrm{i}}}\right) ; \mathrm{i}=1,2$, do not depend on any unknown parameters. So they are pivotal quantities and can be obtained by generating two independent samples from standard exponential distribution, (see, (Thoman et al., 1969)). Using Rlanguage, the following algorithm is used to estimate the generalized confidence interval of $\mathrm{R}$,

\section{Algorithm 3.}

1. Generate two independent random samples $\underline{\mathbf{X}}_{\mathbf{i}}$ from Weibull $\left(\mathrm{b}_{\mathrm{i}}, \mathrm{c}_{\mathrm{i}}\right) ; \mathrm{i}=1,2$, respectively, compute the MLEs $\left(\hat{c}_{1}, \hat{c}_{2}, \hat{b}_{1}, \hat{b}_{2}\right)$ of $\left(c_{1}, c_{2}, b_{1}, b_{2}\right)$.

2. Generate two independent random samples $\underline{\mathbf{X}}_{i}^{*}$ from $\operatorname{Exp}(1) ; i=1,2$, compute the $\operatorname{MLEs}\left(\hat{\mathrm{c}}_{1}^{*}, \hat{\mathrm{c}}_{2}^{*}, \hat{\mathrm{b}}_{1}^{*}, \hat{\mathrm{b}}_{2}^{*}\right)$.

3. Compute the GPQs, $\mathrm{G}_{\mathrm{c}_{\mathrm{i}}}, \mathrm{G}_{\mathrm{b}_{\mathrm{i}}}, \mathrm{G}_{\varphi_{\mathrm{i}}}$, and $\mathrm{G}_{\mathrm{R}} ; \mathrm{i}=1,2$.

4. Repeat the steps $2-3, N$ times to obtain a set of samples of $G_{R}$, say $\left\{G_{R_{j}} ; j=1, \ldots, N\right\}$, and the ordered $G_{R_{j}} ; j=1, \ldots, N$, will be denoted as $\mathrm{G}_{\mathrm{R}_{\mathrm{j}}}^{(1)}<\cdots<\mathrm{G}_{\mathrm{R}_{\mathrm{j}}}^{(\mathrm{N})}$.

5. Construct the $(1-\alpha) 100 \%$ generalized confidence interval of $\mathrm{R}$ as $\left(\mathrm{G}_{\mathrm{R}(\alpha / 2)}, \mathrm{G}_{\mathrm{R}(1-\alpha / 2)}\right)$.

The bootstrap confidence intervals and the Bayesian credible intervals are obtained, using Algorithm 1 and 2, respectively.

\section{Simulation Study}

In this section, we present a simulation study to observe the behavior of the different interval estimators of $\mathrm{P}\left(\mathrm{X}_{1}<\mathrm{X}_{2}\right)$ for different sample sizes and different parameter values for the Weibull distribution. The comparison is based on average length, average coverage, left and right tail errors when $\alpha=0.05$. We generate 1000 samples of sample sizes $\left(\mathrm{n}_{1}, \mathrm{n}_{2}\right)=$ $(10,10)$ and $(30,30)$ from the Weibull distributions of $X_{1}$ and $X_{2}$. We select the parameter values that produce the values of $\mathrm{R}=0.6340,0.7216,0.8011,0.9066,0.9506$, and 0.9707 .

In Bayesian estimation, the hyper-parameters of priors in the two scenarios have the same means but different variances.

1. Let $\left(\alpha_{1}, \beta_{1}\right)=(2,1),\left(\alpha_{2}, \beta_{2}\right)=(1,1 / 2),\left(\delta_{1}, \lambda_{1}\right)=(3,3 / 2)$, and $\left(\delta_{2}, \lambda_{2}\right)=(4,2)$.

2. Let $\left(\alpha_{1}, \beta_{1}\right)=(2,1)$, and $\left(\alpha_{2}, \beta_{2}\right)=(1,1 / 2)$. 


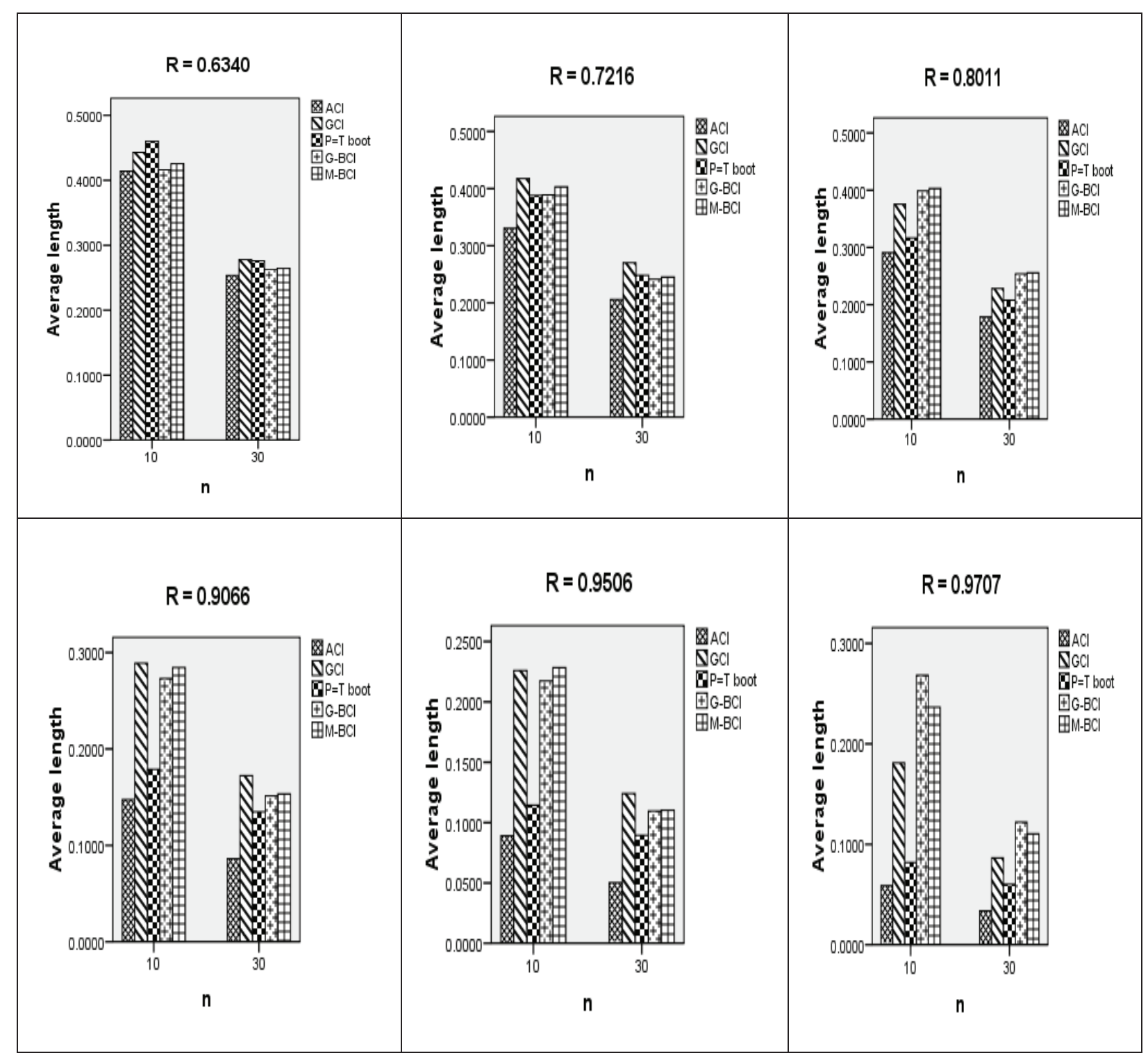

Figure 1. Average length for Weibull distribution 


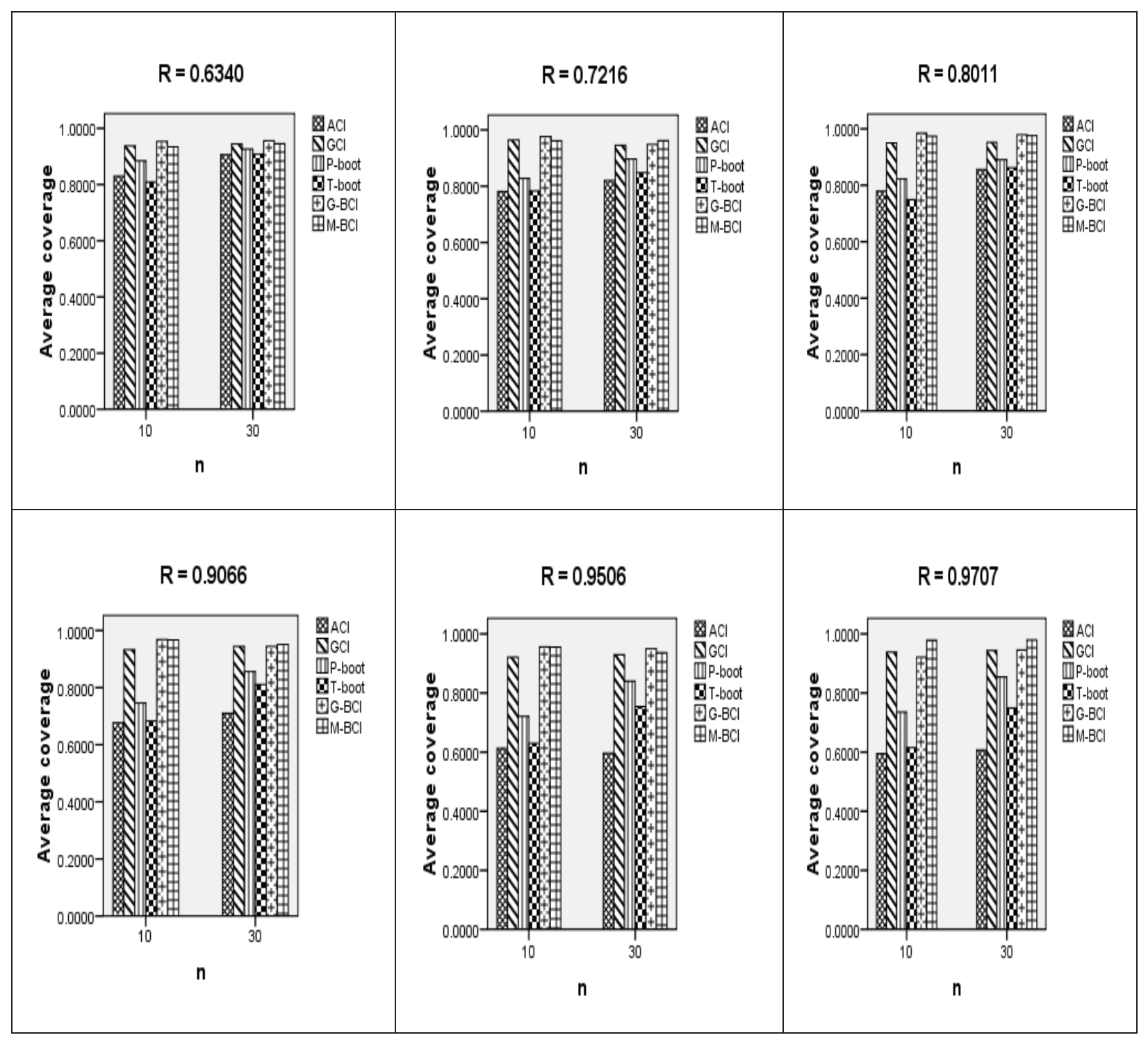

Figure 2. Average coverage probability for Weibull distribution 


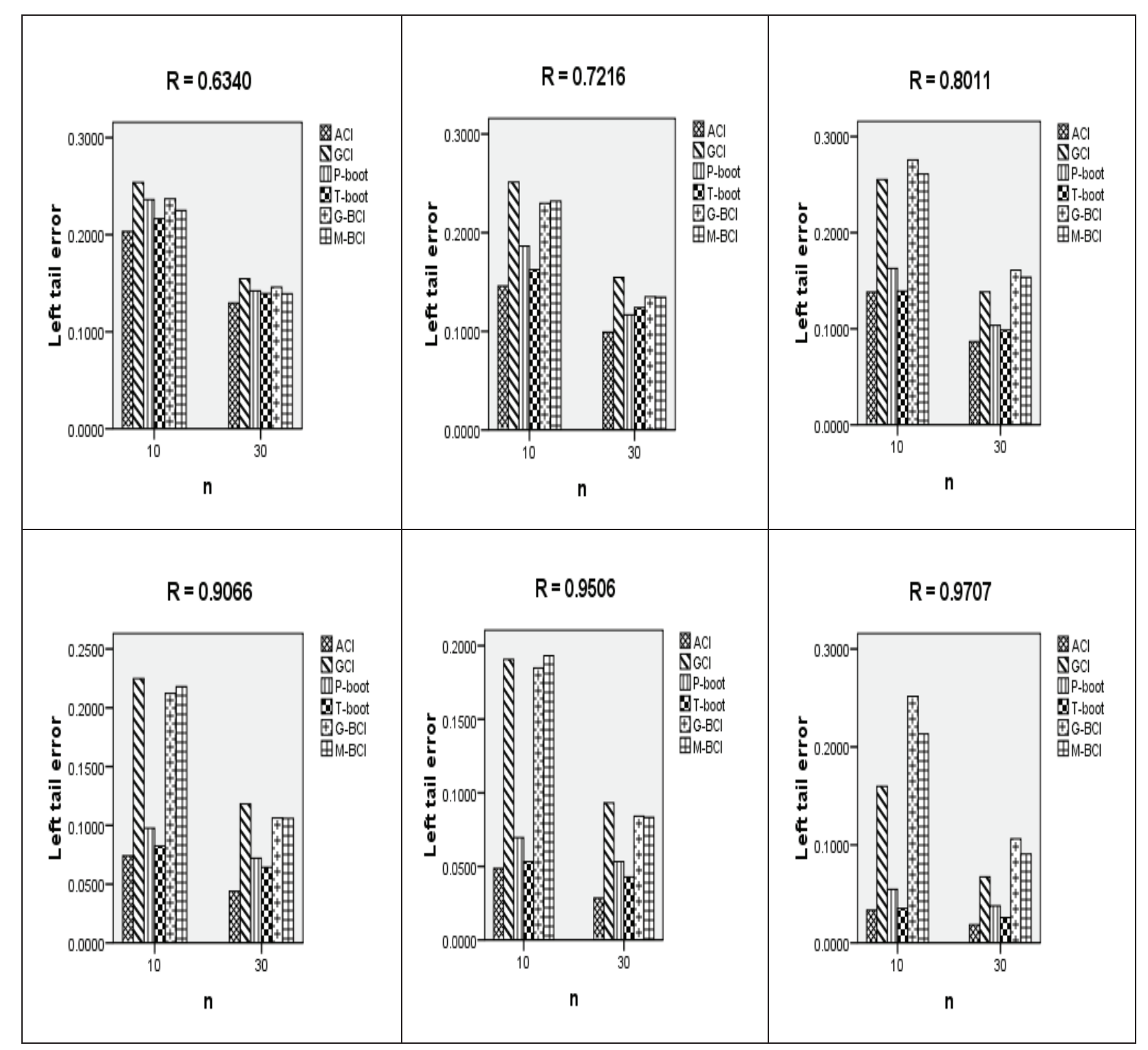

Figure 3. Left tail error for Weibull distribution 


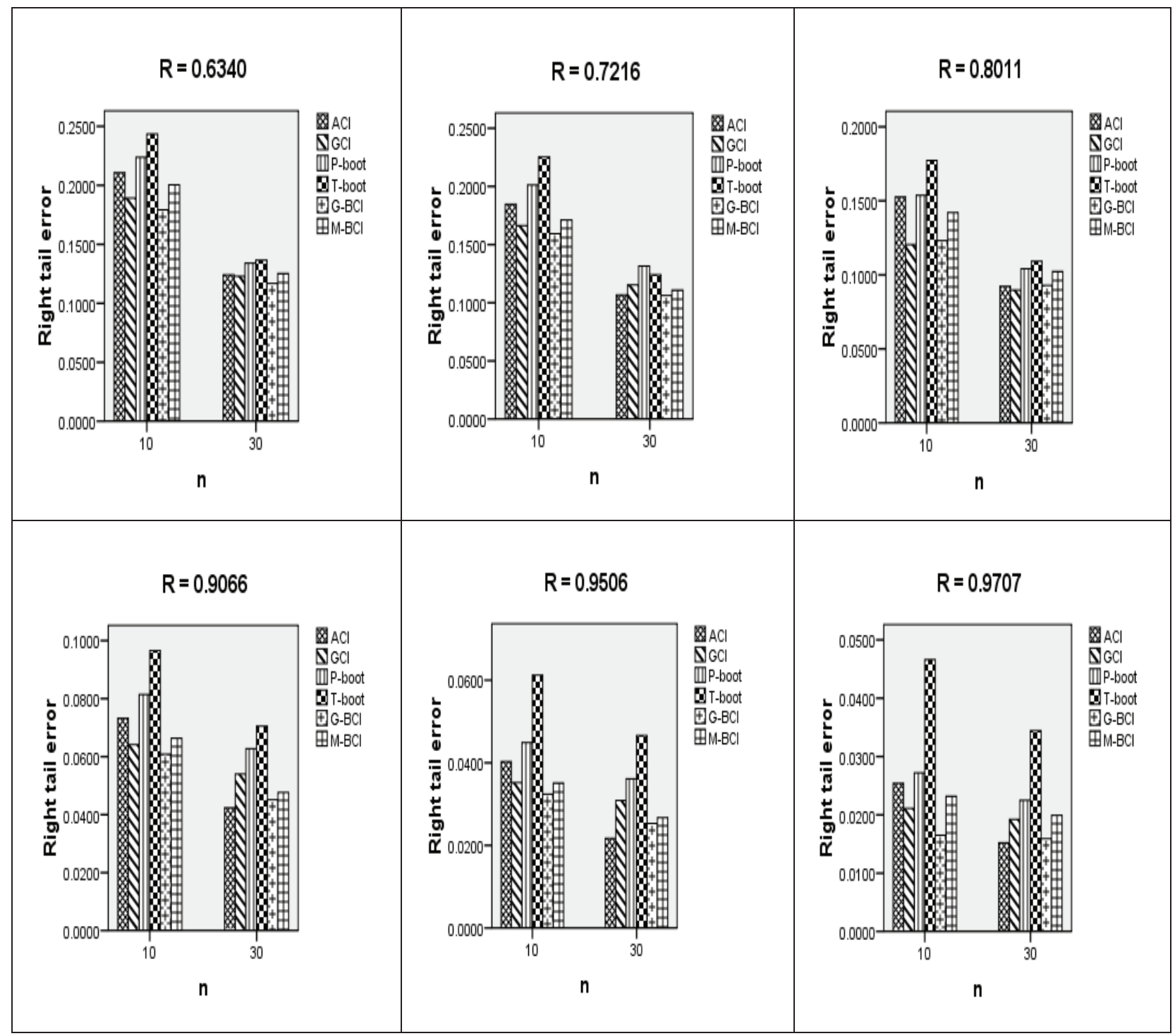

Figure 4. Right tail error for Weibull distribution

In Figure (1), the average length of ACI is the smallest at all the values of R. All lengths of the intervals estimator of $\mathrm{R}$ decrease when $\mathrm{n}$ increases. Lengths of ACI, GCI, and boot decrease when R increases. In Figure (2) we observed that, the average coverage probability of ACI and T-boot are the worst, and the GCI and BCI are roundly approximated $(1-\alpha) 100 \%$, the boot is affected by $\mathrm{n}$ and R. In Figure (3) we see the following; the ACI has the smallest left tail error. All left tail errors of the intervals decrease when $\mathrm{n}$ increases. All left tail errors of the intervals except BCI decrease when $\mathrm{R}$ increases. As we see in Figure (4), the right tail error of boot is the largest and all right tail errors of the intervals are affected by $\mathrm{n}$ and $\mathrm{R}$.

\section{Acknowledgment}

The authors would like to thank the Editor-in-Chief, two anonymous referees for their valuable comments and insightful suggestions on this paper.

\section{References}

Asgharzadeh, A., Kazemi, M., \& Kundu, D. (2017). Estimation of $\mathrm{P}(\mathrm{X}>\mathrm{Y})$ for Weibull Distribution Based on Hybrid Censored Samples,International Journal of Systems Assurance Engineering and Management, 8, 489-498.

https://doi.org/10.1007/s13198-015-0390-2 
Asgharzadeh, A., Valiollahi, R., \& Raqab, M. Z. (2011). Stress-Strength Reliability of Weibull Distribution Based on Progressively Censored Samples, Statistics and Operations Research Transactions, 35, 103-124.

Asgharzadeh, A., Valiollahi, R. \& Raqab, M. Z. (2013). Estimation of the Stress-Strength Reliability for the Generalized Logistic Distribution, Statistical Methodology, 15, 73-94. https://doi.org/10.1016/j.stamet.2013.05.002

Birnbaum, Z., \& McCarty, R. (1958). A Distribution-Free Upper Confidence Bound for $\mathrm{P}(\mathrm{Y}<\mathrm{X})$ Based on Independent Samples of X and Y, Annals of Mathematical Statistics, 29, 558-562. https://doi.org/10.1214/aoms/1177706631

Birnbaum, Z. (1956). On a Use of Mann-Whitney Statistics, Statistics and Probability, University of California Press.

Efron, B. (1994). An Introduction to the Bootstrap, Chapman \& Hall.

Kotz, S., Lumelskii, Y., \& Pensky, M. (2003). The Stress-Strength Model and its Generalizations: Theory and Applications, Singapore: World Scientific. https://doi.org/10.1142/5015

Krishnamoorthy, K., \& Lin, Y. (2010). Confidence Limits for Stress-Strength Reliability Involving Weibull Models, Statistical Planning and Inference, 140, 1754-1764. https://doi.org/10.1016/j.jspi.2009.12.028

Krishnamoorthy, K., Lin, Y., \& Xia, Y. (2009). Confidence Limits and Prediction Limits for a Weibull Distribution Based on the Generalized Variable Approach, Statistical Planning and Inference, 139, 2675 - 2684. https://doi.org/10.1016/j.jspi.2008.12.010

Kundu, D., \& Gupta, R. D. (2006). Estimation of $\mathrm{P}(\mathrm{Y}<\mathrm{X})$ for Weibull Distribution, IEEE Transactions on Reliability, 55, 270-280. https://doi.org/10.1109/TR.2006.874918

Mokhlis, N., Ibrahim, E., \& Gharieb, D. (2017). Stress-Strength Reliability with General Form Distributions, Communications in Statistics-Theory and Methods, 46, 1230-1246. https://doi.org/10.1080/03610926.2015.1014110

Panahi, H., \& Asadi, S. (2010). Estimation of $\mathrm{R}=\mathrm{P}(\mathrm{Y}<\mathrm{X})$ for Two-Parameter Burr Type XII Distribution, World Academy of Science, Engineering and Technology International Journal of Mathematical, Computational, Physical, Electrical and Computer Engineering, 12, 2010.

Rao, C. (1965). Linear Statistical Inference and its Applications, New York: John Wiley and Sons.

Saracoglu, B., Kaya, M. F., \& Abd-Elfattah, A. M. (2009). Comparsion of Estimators for Stress-Strength Reliability in the Gompertz Case, Hacettepe Journal of Mathematics and Statistics, 38, 339-349.

Singh, S. K., Singh, U., Yadav, A. S., \& Vishwkarma, P. K. (2015). On the Estimation of Stress-Strength Reliability Parameter of Inverted Exponential Distribution, International Journal of Scientific World, 3, 98-112. https://doi.org/10.14419/ijsw.v3i1.4329

Thoman, D. R., Bain, L. J., \& Antle, C. E. (1969). Inferences on the Parameters of the Weibull Distribution, Technometrics, 11, 445-446. https://doi.org/10.1080/00401706.1969.10490706

\section{Copyrights}

Copyright for this article is retained by the author(s), with first publication rights granted to the journal.

This is an open-access article distributed under the terms and conditions of the Creative Commons Attribution license (http://creativecommons.org/licenses/by/4.0/). 